\title{
DIFFERENTIATION OF ZYGMUND FUNCTIONS
}

\author{
DAVID C. ULLRICH
}

(Communicated by J. Marshall Ash)

\begin{abstract}
The "little- $O$ Zygmund class" $\lambda^{*}$ contains a nowhere-differentiable function.
\end{abstract}

\section{INTRODUCTION}

A classical result due originally to Rajchman and then improved by Zygmund [ZY, p. 43] states that if $f \in \lambda^{*}(T)$ and $f$ is real valued then $f$ must be differentiable on a dense subset of $T$. This implies that if $F \in \mathfrak{B}_{o}$ (the "little$o$ Bloch space") then $\operatorname{Re}(F)$ must possess a radial (and hence nontangential) limit at each point of a dense subset of the boundary.

Somewhat more recently, it was shown [GHP, Theorem 2] that $F$ itself must have a radial limit at each point of a dense subset of the boundary, if $F \in \mathfrak{B}_{o}$. As noted in [GHP], this would follow from the result of Rajchman and Zygmund if the latter were true for a general (complex-valued) element of $\lambda^{*}$, but this question has been open. In this note we show that there exists an $f \in \lambda^{*}$ that is nowhere differentiable and that, in fact, satisfies a Hölder condition of order one at no point.

It turns out that the existence of a nowhere differentiable $f \in \lambda^{*}$ is also one of various results in [MAK2], including the fact that if $f \in \lambda^{*}$ and is either realvalued or extends to a function holomorphic in the disc then the set of points where $f$ is differentiable must have Hausdorff dimension 1. (The results in [MAK2] are proved in more detail in [MAK1], in particular, cf. [MAK1, Theorem 5.5].) It seems that the extremely simple argument below may nonetheless be of some independent interest: If $u$ is an appropriate (real-valued) lacunary trigonometric series then $u \in \lambda^{*}$ and $u$ is differentiable only on a set of measure zero. Now one may construct $v \in \lambda^{*}$ so that $f=u+i v$ is nowhere differentiable (in particular, we do not require the main technical device in [MAK2]-a characterization of the dyadic martingales arising from elements of $\lambda^{*}$ ).

\section{THEOREM}

The notation $\lambda^{*}(T)$ refers to the "little-o" Zygmund class on the unit circle $T$ : we write $f \in \lambda^{*}(T)$ if $f$ is a continuous (complex-valued) function on $T$

Received by the editors May 24, 1991.

1991 Mathematics Subject Classification. Primary 42A55. 
and

$$
\lim _{h \rightarrow 0} h^{-1}\left|f\left(e^{i(t-h)}\right)-2 f\left(e^{i t}\right)+f\left(e^{i(t+h)}\right)\right|=0,
$$

uniformly in $t$ (the functions in $\lambda^{*}$ are called "smooth functions" in [ZY]). We set

$$
M f(t)=\sup _{h>0} h^{-1}\left|f\left(e^{i(t+h)}\right)-f\left(e^{i t}\right)\right|,
$$

so that $f$ satisfies a Hölder condition of order 1 at $e^{i t}$ if and only if $M f(t)<$ $\infty$.

Theorem. There exists $f \in \lambda^{*}(T)$ such that $M f(t) \equiv x$.

We will set $f=u+i v$, where $u \in \lambda^{*}$ is a (real-valued) lacunary series with $M u=\propto$ a.e. It is impossible to achieve $M u \equiv \propto$ here, but the following proposition will provide us with a real-valued function $v \in \lambda^{*}$ such that $M v=$ $\infty$ at every point of the set where $M u<x$.

Proposition. Suppose $E \subset T$ is an $F_{\sigma}$ of (Lebesgue) measure zero. Then there exists a real-valued $v \in \lambda^{*}(T)$ such that $(d / d t) v\left(e^{i t}\right)=\infty$ for every $t \in E$.

This will follow from the following lemma. The notation $\operatorname{VMO}(T)$ refers to the space of functions of vanishing mean oscillation, as usual.

Lemma. Suppose $E$ is as in the proposition. There exists $\varphi \in \operatorname{VMO}(T)$ such that $\varphi \geq 0$ on $T$ and $\lim _{s \rightarrow t} \varphi\left(e^{i s}\right)=\propto$ for every $e^{i t} \in E$.

Proof. If we can prove the lemma for compact $E$ then the general case follows because $\varphi \geq 0$. Suppose $E \subset T$ is a compact set of measure zero.

This implies that $E$ is a peak set for the disc algebra: there exists a function $g$ that is holomorphic in the unit disc $D$ and continuous on $\bar{D}$, such that $g\left(e^{i t}\right)=1$ for $e^{i t} \in E$, while $|g(z)|<1$ for $z \in \bar{D} \backslash E$.

Now let $\Omega=\{x+i y: x>1,|y|<1 / x\}$ and let $\psi: D \rightarrow \Omega$ be holomorphic and surjective. A theorem of Carathéodory shows that $\psi$ extends to a homeomorphism $\bar{\psi}: \bar{D} \rightarrow \bar{\Omega}$, where $\bar{\Omega}$ denotes the closure of $\Omega$ on $S$, the Riemann sphere; we may take $\bar{\psi}(1)=\propto$.

Thus $G=\bar{\psi} \circ g: \bar{D} \rightarrow S$ is continuous. Let $\varphi=\operatorname{Re}(G)$. Then $\varphi$ (restricted to $T)$ is a continuous map from $T$ to $[0, \infty]$ such that $\varphi\left(e^{i t}\right)=\propto$ for $e^{i t} \in E$. We only need to show that $\varphi \in \mathrm{VMO}$, but $\varphi \in \mathrm{VMO}$ because $\varphi$ is the harmonic conjugate of a continuous function: The point to our choice of $\Omega$ was that $\operatorname{Im}(z) \rightarrow 0$ as $z$ tends to $\propto$ within $\Omega$, and this shows that $\operatorname{Im}(G) \in C(T)$.

Proof of the proposition. Given an $F_{\sigma}$ set $E \subset T$ of measure zero, choose $\varphi$ as in the lemma. Now define $\varphi_{1}=\varphi-c$, where $c=(2 \pi)^{-1} \int_{0}^{2 \pi} \varphi\left(e^{i t}\right) d t$, and let $v$ be an absolutely continuous function such that $(d / d t) v\left(e^{i t}\right)=\varphi_{1}\left(e^{i t}\right)$ almost everywhere. It follows that $(d / d t) v\left(e^{i t}\right)=\propto$ for $t \in E$, while the fact that $\varphi \in \mathrm{VMO}$ implies that $v \in \lambda^{*}$. 
Proof of the theorem. Choose a sequence $a_{j} \geq 0$ with $\lim _{j \rightarrow \infty} a_{j}=0$ but $\sum_{j=1}^{\infty} a_{j}^{2}=\infty$, and set

$$
u\left(e^{i t}\right)=\sum_{j=1}^{\infty} 2^{-j} a_{j} \cos \left(2^{j} t\right) .
$$

Now the fact that $a_{j} \rightarrow 0$ shows that $u \in \lambda^{*}$ [ZY, Theorem 4.10, p. 47], while $\sum_{j=1}^{\infty} a_{j}^{2}=\infty$ shows that $M u\left(e^{i t}\right)=\infty$ for almost all $t$. This will be "clear" to readers with some experience dealing with lacunary series; a proof is already at least implicit in [ZY]:

Let $d_{N}(t)=-\sum_{j=1}^{N} a_{j} \sin \left(2^{j} t\right)$. Then it is well known that $\left(d_{N}(t)\right)$ is unbounded for almost every value of $t$ [ZY, Theorem 6.4, p. 203 and Remark (c), p. 205]. But it is easy to obtain a uniform upper bound on the quantity

$$
h_{N}^{-1}\left[u\left(e^{i\left(t+h_{N}\right)}\right)-u\left(e^{i t}\right)\right]-d_{N}(t)
$$

if $h_{N}=2^{-N} \pi$, so that $M u=\infty$ at any point where $\left(d_{N}\right)$ is unbounded.

Now let $E=\left\{e^{i t}: M u\left(e^{i t}\right)<\infty\right\}$. We have just seen that $E$ has measure zero. Continuity of $u \in \lambda^{*}$ shows that $\{M u \leqslant j\}$ is closed for $j=1,2, \ldots$, so that $E$ is an $F_{\sigma}$. Choose $v$ as in the proposition and let $f=u+i v$. Then $f \in \lambda^{*}$ and $M f \equiv \infty$.

\section{REFERENCES}

[GHP] D. Gnuschke-Hauschild and Ch. Pommerenke, On Bloch functions and gap series, J. Reine Angew. Math. 367 (1986), 172-186.

[MAK1] N. G. Makarov, Probability methods in conformal mappings. II, LOMI preprint E-14-88, Leningrad, 1988.

[MAK2] _ On the radial behavior of Bloch functions, Soviet Math. Dokl. 40 (1990), 505-507.

[ZY] A. Zygmund, Trigonometric series, 2nd ed., vol. 1, Cambridge Univ. Press, Cambridge, 1959.

Department of Mathematics, Oklahoma State University, Stillwater, Oklahoma 74078-0001

E-mail address: ULLRICH@HARDY.MATH.OKSTATE.EDU 